\title{
Preparation of Gelatin/Polycaprolactone Electrospun Fibers Loaded with Cis-Platinum and Their Potential Application for the Treatment of Prostate Cancer
}

\author{
Yang Jin, ${ }^{1}$ Peng Sun, ${ }^{2}$ Tong Wu, ${ }^{3}$ Jin Wang, ${ }^{4}$ Xiaoyu Huang, ${ }^{5}$ Shishuai Zuo, ${ }^{2}$ Zilian Cui, ${ }^{2,5}$ \\ Ji Chen, ${ }^{2}$ Lianjun Li, ${ }^{2}$ Ning Suo, ${ }^{2}$ Xunbo Jin, ${ }^{2,5}$ and Dong Zhang ${ }^{2,5}$ \\ ${ }^{1}$ Department of Anesthesiology, Shandong Provincial Hospital, Cheeloo College of Medicine, Shandong University, Jinan, \\ Shandong 250021, China \\ ${ }^{2}$ Department of Urology, Shandong Provincial Hospital Affiliated to Shandong First Medical University, Jinan, \\ Shandong 250021, China \\ ${ }^{3}$ Department of Chemotherapy, Shandong Second Provincial General Hospital, Shandong Provincial ENT Hospital, Jinan, \\ Shandong 250021, China \\ ${ }^{4}$ Department of Urology, The First Affiliated Hospital of Shandong First Medical University, Jinan, Shandong 250021, China \\ ${ }^{5}$ Department of Urology, Shandong Provincial Hospital, Cheeloo College of Medicine, Shandong University, Jinan, \\ Shandong 250021, China
}

Correspondence should be addressed to Dong Zhang; zd_sdu@163.com

Received 9 March 2021; Revised 31 March 2021; Accepted 7 April 2021; Published 22 April 2021

Academic Editor: Songwen Tan

Copyright ( 2021 Yang Jin et al. This is an open access article distributed under the Creative Commons Attribution License, which permits unrestricted use, distribution, and reproduction in any medium, provided the original work is properly cited.

\begin{abstract}
Although the development of nanomaterials for cancer therapy has received much attention in recent years, prostate cancer still remains one of the most troubling cancers in males. In this work, biomimetic polycaprolactone (PCL)/gelatin (GT)/cis-platinum (CDDP) (PG@CDDP) fibrous films were developed via electrospinning technology. The microstructure and chemical properties of PG@CDDP fibers are investigated. It is found that the microscopic morphology and diameter of PG@CDDP fibers are changed compared to those of the PG fibers. The Fourier transform-infrared spectroscopy and wide-angle X-ray diffraction demonstrate that CDDP is successfully incorporated into the fibers. The human prostate cancer cells in vitro to electrospun films (PG and PG@CDDP) were evaluated based on initial cell response and cell viability. The density, elongation, and viability of adhered cancer cells significantly reduce with an increased concentration of CDDP in PG fibers. Thus, the developed PG@CDDP fiber matrix has great potential as candidate scaffolds for the treatment of prostate cancer.
\end{abstract}

\section{Introduction}

Cancer has been the main cause of human death for centuries due to the uncontrolled proliferation of cancer cells as well as tumor metastasis [1-3]. Particularly, prostate cancer is a common malignant tumor in males and has been regarded as a global issue in developing and underdeveloped countries $[4,5]$. Generally, prostate cancer can be described as adenocarcinoma, but it can develop into other histopathological types, e.g., small-cell and neuroendocrine carcinoma $[4,6]$. Traditional chemotherapy (e.g., the combination of docetaxel (Taxotere) and prednisone) and radiotherapy are still the most commonly used strategy in clinical cancer treatment $[7,8]$. However, both strategies had some limitations, such as serious side effects, limited therapeutic efficacy, multidrug resistance, and poor efficacy for metastatic tumors [8-11].

Recently, micro-/nanomaterials have gained increasing attention for the treatment of cancer owing to enhanced therapeutic efficiency and relatively mild systemic side effect $[8,11,12]$. In particular, micro-/nanofibers as a bioscaffold have been designed as promising carriers for delivering anticancer drugs because of a high loading capacity and high encapsulation efficiency [13-15]. While numerous micro/nanofabrication strategies, e.g., phase separation, synthetic 


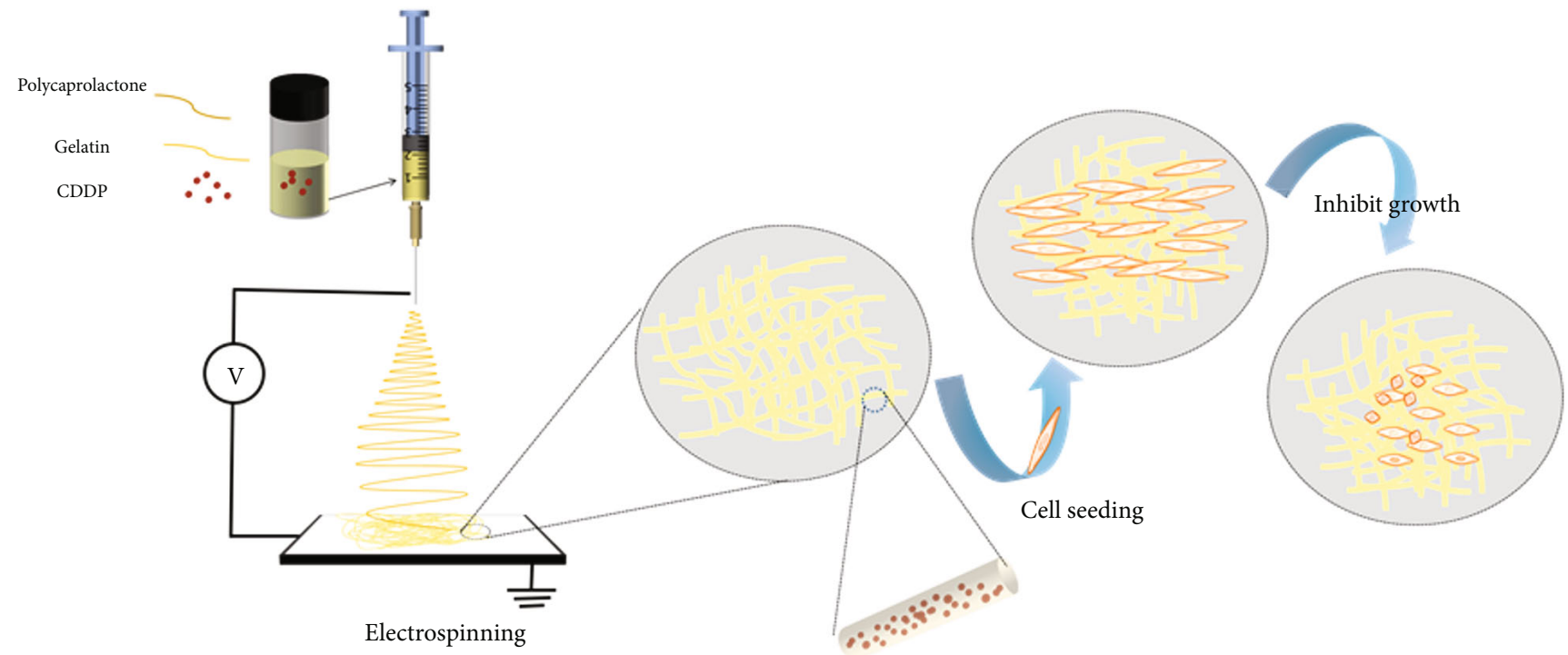

FIGURE 1: Schematic diagram of the preparation of PG@CDDP fibers and their interaction with prostate cancer cells.

molding, self-assembly, and drawing, have provided useful tools to prepare ultrafine fiber, in the past decade, electrospinning has received more interest in developing ultrafine polymer fibers for drug delivery applications owing to their extracellular matrix- (ECM-) like structure, excellent biological properties, high mechanical strength, porous mesh with remarkable interconnectivity, high specific surface area, and aspect ratio [16-19]. Electrospun fibrous films can be conveniently implanted into the location of the tumor for the controlled release of chemotherapeutics and ultimately achieve the purpose of curing cancer [20,21].

Gelatin/polycaprolactone fibers are selected in this study as an anticancer drug delivery system because both polymers possess excellent biocompatible, tunable biodegradable, and nontoxic degradation products $[16,22,23]$. It does not require surgical removal after the completion of drug release. Anticancer drug (cis-platinum) has been widely used as a chemotherapy medication to treat many cancers [24, 25]. In the present work, biodegradable polycaprolactone (PCL)/gelatin (GT)/cis-platinum (CDDP) (PG@CDDP) fibrous films were fabricated via electrospinning technology. The morphology and chemical properties of PG@CDDP fibers were investigated by scanning electron microscopy (SEM) and Fourier transform infrared (FTIR) and X-ray diffraction (XRD) spectroscopy. The human prostate cancer cells (T24) in vitro that interacted with electrospun fiber films were tested.

\section{Experimental Section}

2.1. Materials. Cis-platinum (CDDP) was purchased from Macklin (Shanghai, China). Polycaprolactone (PCL) was bought from Aladdin (Shanghai, China). Gelatin (GT) and trifluoroethanol (TFE) were obtained from Sigma-Aldrich (USA). All reagents were of analytical grade and used without further purification. Deionized (DI) water in all experiments was used.
2.2. Preparation of GT/PCL Solutions. Solutions of PCL/GT (PG) were obtained by mixing $10 \%(w / v)$ PCL/TFE and $10 \%(w / v)$ GT/TFE at the mass ratio of $1: 1$ and under stirring for $24 \mathrm{~h}$. To make miscible and transparent PG/TFE solutions, $10 \mu \mathrm{L}$ acetic acid was added to the PG solutions according to previous studies [23]. Next, a certain amount of CDDP (i.e., $0.05 \mathrm{~g}$ and $0.1 \mathrm{~g}$ ) was introduced into PG solutions to prepare $10 \mathrm{~mL}$ PG@0.5\%CDDP and PG@1\%CDDP solutions, respectively.

2.3. Electrospinning. Briefly, solutions of PG@CDDP in a $5 \mathrm{~mL}$ syringe were delivered at a feeding rate of $1.0 \mathrm{~mL} / \mathrm{h}$ by a syringe pump (Langer, Baoding, China). A high-voltage power supply (Dongwen, Tianjin, China) of $12.5 \mathrm{kV}$ was used between a blunt metal needle tip and a collector, separated at a distance of $14 \mathrm{~cm}$. All the electrospinning processes were performed on a perpendicular electrospinning strategy at room temperature $\left(21-23^{\circ} \mathrm{C}\right)$ with an ambient humidity of $45-50 \%$.

2.4. Scanning Electron Microscopy (SEM). The morphology of PG composite fibers was observed using SEM (VEGA3, TESCAN, Czech). Before imaging, samples were sputter-coated with gold to increase conductivity. The average diameter of PG composite fibers was analyzed by detecting at least 50 fiber segments using ImageJ.

2.5. Fourier Transform Infrared (FTIR) Spectroscopy. A Nicolet iN10 FTIR spectrometer (Thermo Fisher Scientific, Waltham, MA, USA) was applied to detect the FTIR spectra of PG composite fibers over the range of $500-4000 \mathrm{~cm}^{-1}$ at a scanning resolution of $2 \mathrm{~cm}^{-1}$ during 32 scans.

2.6. X-Ray Diffraction (XRD) Spectroscopy. XRD spectroscopy was carried out to detect the crystal structures of PG composite fibers. The materials were measured between 5 and $80^{\circ}(2 \theta)$ at a scanning rate of $1.0^{\circ}(2 \theta)$ per minute 

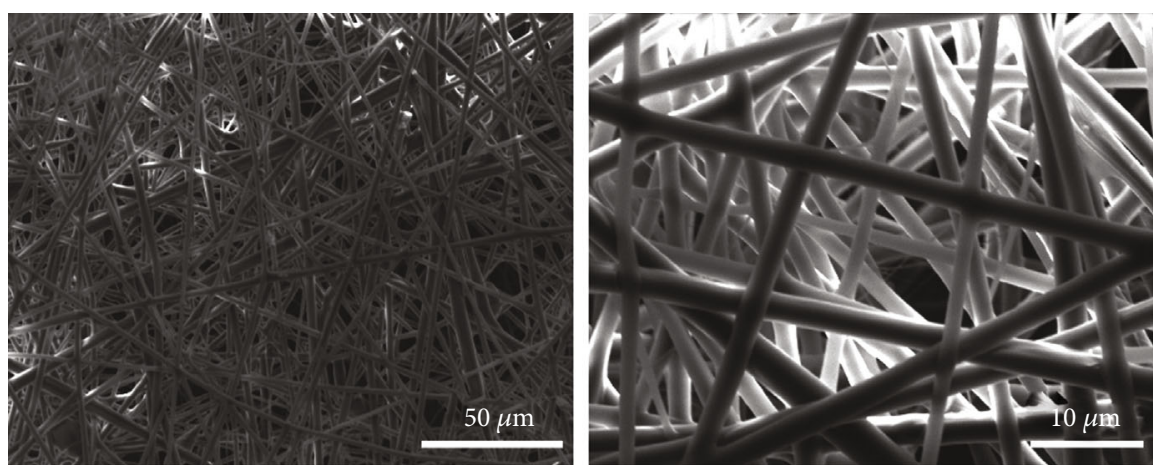

(a)
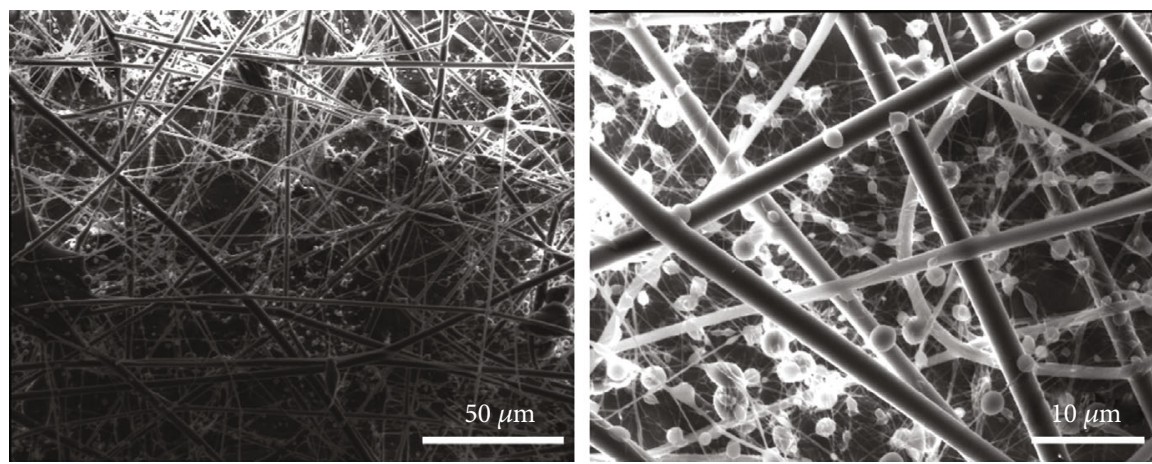

(b)
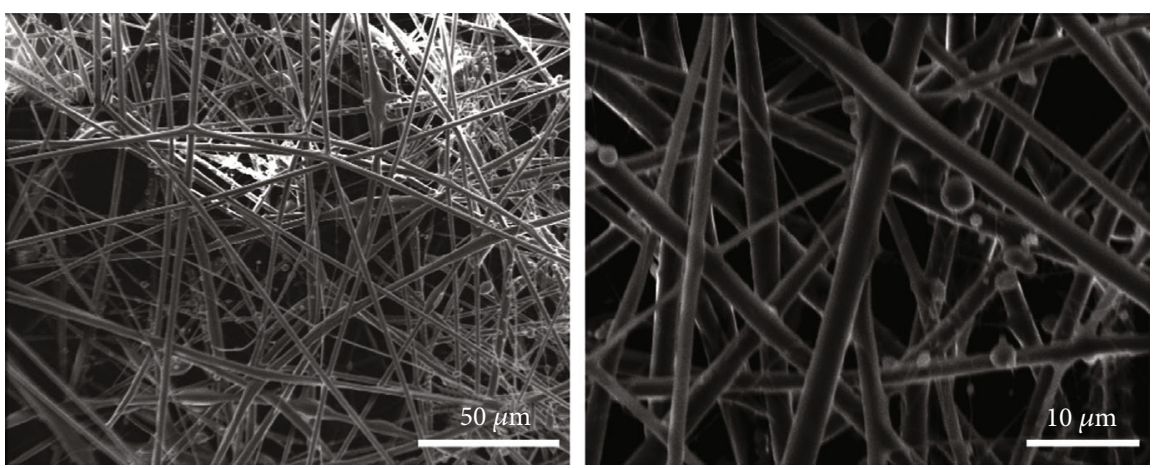

(c)
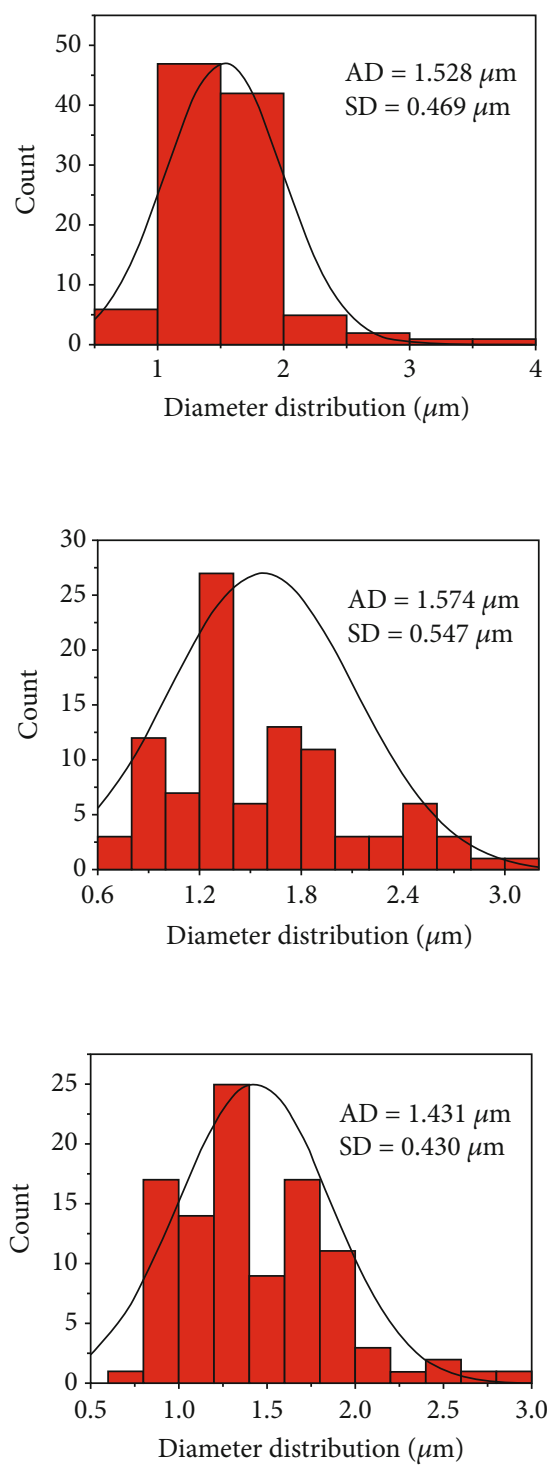

Figure 2: SEM images and fiber diameter distribution of the electrospun fibers of (a) PG, (b) PG@0.5\%CDDP, and (c) PG@1\%CDDP.

operating with voltage $40 \mathrm{kV}$ and current $30 \mathrm{~mA}$ equipped by $\mathrm{Cu} \mathrm{K} \alpha$ radiation.

2.7. In Vitro Drug Release. $40 \mathrm{mg}$ of PG@0.5\%CDDP or PG@1.5\%CDDP nanofibers was added in $3 \mathrm{~mL}$ of normal saline $(0.9 \% \mathrm{NaCl})$ and incubated at $37^{\circ} \mathrm{C}$ in a shaker. At predetermined time intervals, $1 \mathrm{~mL}$ aliquots of solution were removed for the measurement and replaced with $1 \mathrm{~mL}$ fresh buffer solution. Aliquots of solution were detected using a High-Performance Liquid Chromatography (HPLC) system (Shimadzu, Kyoto, Japan).

\subsection{Cell Assays}

2.8.1. Cell Culture. Human prostate cancer cells (T24) were purchased from Biyuntian Biological Technology Co., Ltd. (Shanghai, China). Cells were cultured and expanded in
RPMI-1640 supplemented with 10\% FBS (Biological Industries, Israel) at $37^{\circ} \mathrm{C}$ with $5 \% \mathrm{CO}_{2}$ in the air. The cells were harvested at $80-90 \%$ confluency from culture flasks by tryp$\sin$ at $37^{\circ} \mathrm{C}$ for further subcultures.

2.8.2. Fluorescence Staining. Cancer cells were seeded in 24well plates at a density of $5.0 \times 10^{4}$ cells/well for $1 \mathrm{~d}$. The cells were fixed with $4 \%$ paraformaldehyde for $15 \mathrm{~min}$, rinsed three times with PBS, and then permeabilized with $0.1 \% \mathrm{X}$ 100 for $6 \mathrm{~min}$. The fixed cells were washed twice with PBS and blocked with $5 \%$ bovine serum albumin (BSA) in PBS for $40 \mathrm{~min}$. The cell cytoskeleton and nuclei were labeled with TRITC-phalloidin and 4',6-diamidino-2-phenylindole (DAPI) for $20 \mathrm{~min}$. Cell images were obtained using a fluorescent microscope. The elongation factor of the cell was analyzed as the major axis/minor axis of the cells. By definition, the roundness is equal to 1 for a completely round cell. 


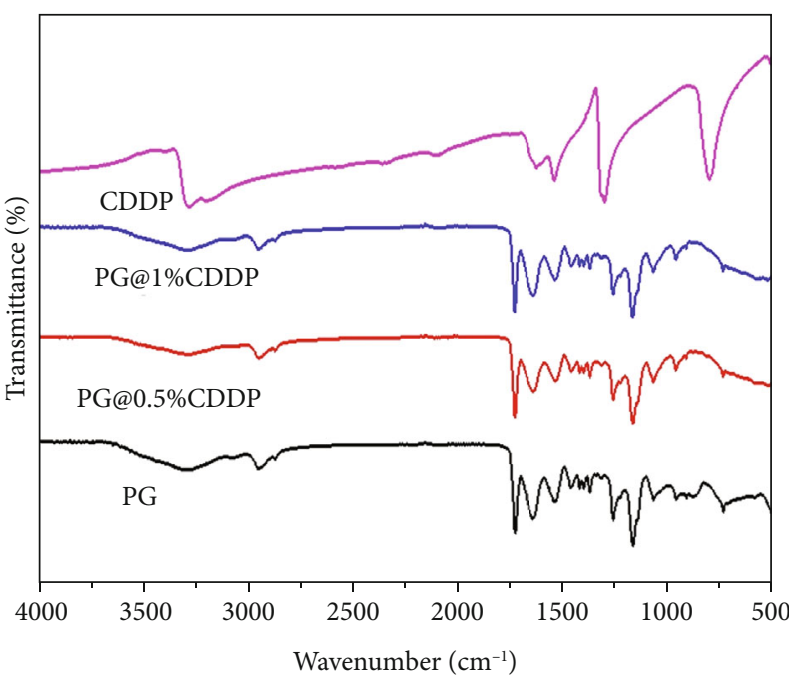

(a)

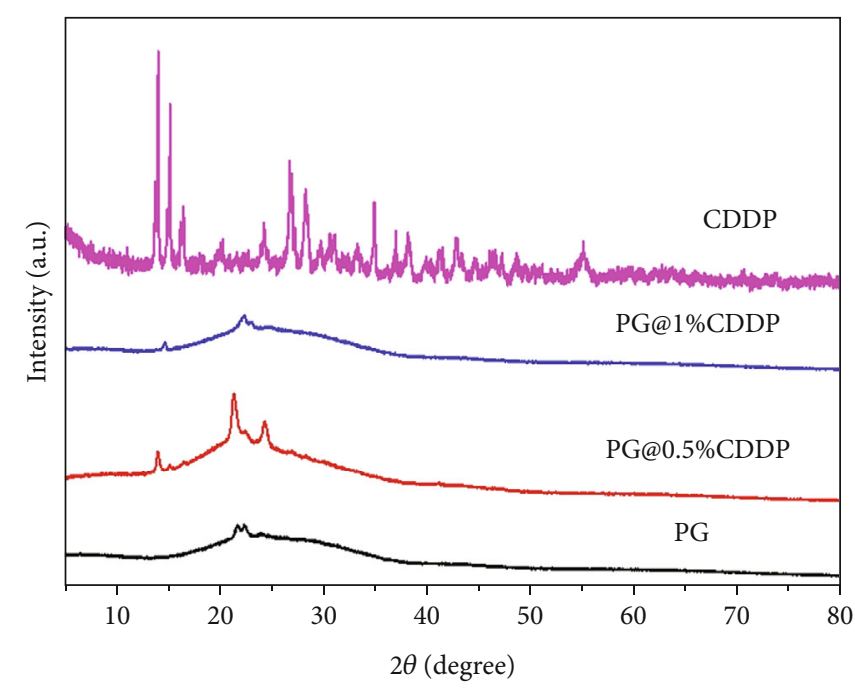

(b)

FIgURe 3: (a) FTIR spectra and (b) XRD patterns of CDDP, PG, PG@0.5\%CDDP, and PG@1\%CDDP fibers.

2.8.3. Cell Viability. Cancer cells were seeded in 24-well plates at a density of $3.0 \times 10^{3}$ cells/well for 1,3 , and $5 \mathrm{~d}$. Next, the medium was exchanged with a fresh medium containing 10\% Cell Counting Kit-8 (Absin Bioscience Inc., China) and incubated for $2 \mathrm{~h}$. The optical density (OD) values of the medium were measured at $450 \mathrm{~nm}$ using a microplate reader.

2.9. Statistical Analysis. All values were shown as mean \pm standard deviation (SD). Statistical analysis was performed using Origin 9. One-way analysis of variance (ANOVA) with Tukey's test was used to identify differences between groups. A value of $p<0.05$ was considered to be statistically significant.

\section{Results and Discussion}

3.1. Preparation and Characterization of Composite Fibers. The preparation process of PG@CDDP fibers and their interaction with prostate cancer cells are illustrated in Figure 1. The electrospun GT/PCL couple is one of the most intensively investigated natural-synthetic hybrid fiber systems owing to its excellent electrospinnability, ECM-like structure, favorable biological properties, sufficient mechanical strength, and capability for drug loading.

The morphology of nanofibers plays a critical role in their (bio)physicochemical properties, such as mechanical strength, biodegradation, and cell responses [26-29]. The morphology of composite fibers was observed by SEM (Figure 2). As shown in Figure 2(a), the prepared fibers were highly uniform and smooth with an averaged diameter of $1.528 \pm 0.469 \mathrm{~nm}$. When $0.5 \% \mathrm{CDDP}$ was added into the PG system, there was no significant difference in the fiber diameter between PG@0.5\%CDDP fibers and PG fibers (Figure 2(b)). However, the microstructure of PG@0.5\%CDDP fibers greatly changed compared to that of the PG fibers. And small particles among the fibers were found in the PG@0.5\%CDDP system, probably due to the formation of phase separation after the

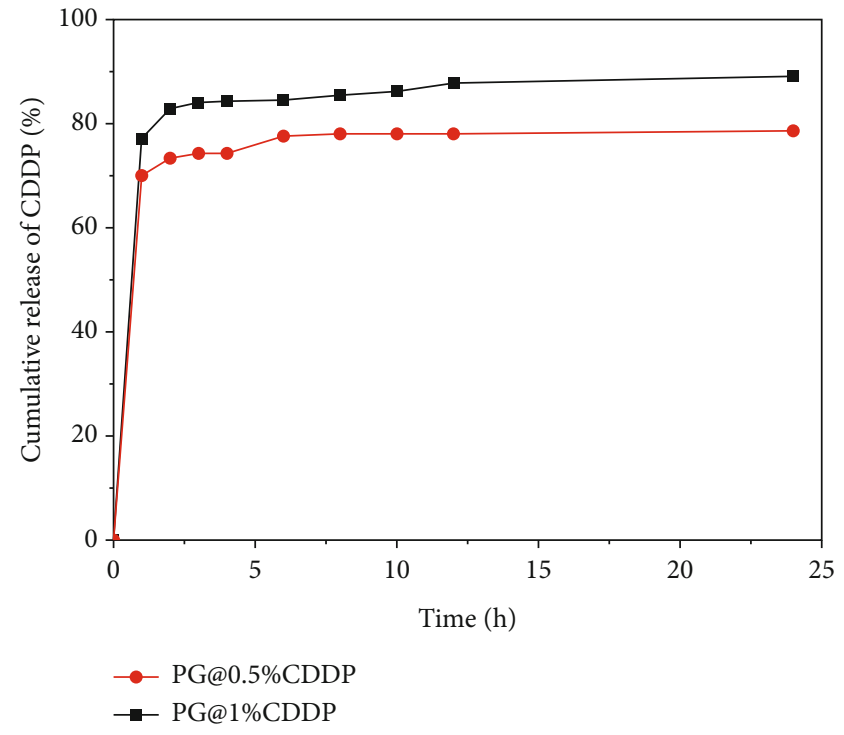

FIGURE 4: In vitro release of CDDP from PG@0.5\%CDDP and PG@1\%CDDP fibers.

addition of CDDP. With an increased amount of CDDP, the diameter of PG@1\%CDDP fibers slightly reduced as compared to those of the PG and PG@0.5\%CDDP fibers. Small particles among the PG@1\%CDDP fibers significantly decreased compared to those among the PG@0.5\%CDDP system. Taken together, these results indicate that the microstructure of $\mathrm{PG}$ fibers was dependent on the introduction of CDDP.

The chemical groups of PG-based composite fibers were analyzed by FT-IR. Figure 2(a) shows the FT-IR spectra of CDDP, PG fibers, PG@0.5\%CDDP fibers, and PG@1\%CDDP fibers. In the CDDP group, the typical band at $3200-3283 \mathrm{~cm}^{-1}$ belonged to the asymmetric and symmetric tensile vibrations of $\mathrm{N}-\mathrm{H}$; the typical band at $1300-1618 \mathrm{~cm}^{-1}$ was assigned to the symmetric and asymmetric bending vibrations of $\mathrm{H}-\mathrm{N}-\mathrm{H}$; the peak at $800 \mathrm{~cm}^{-1}$ corresponded to the tensile vibration signal of N-Pt [30]. 

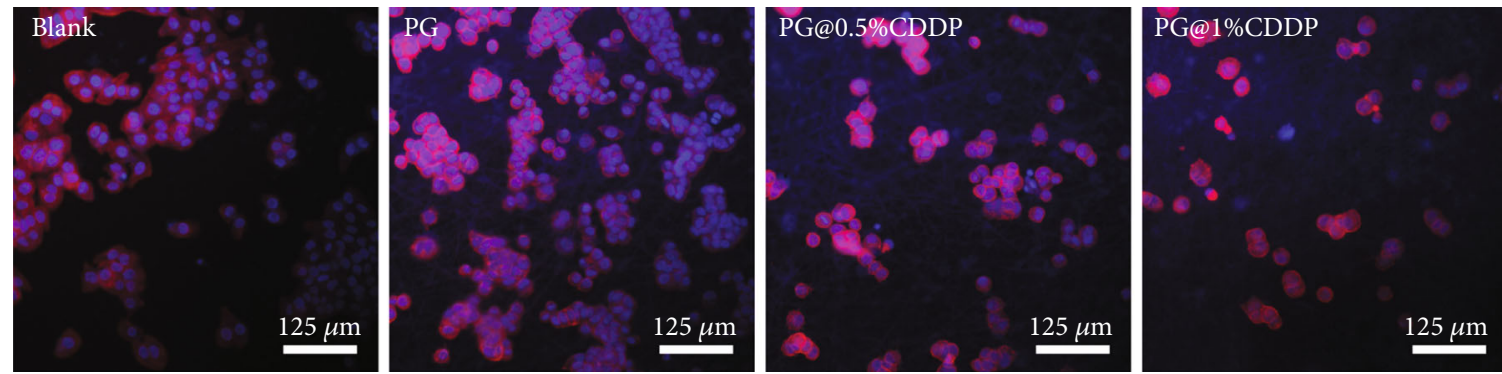

(a)

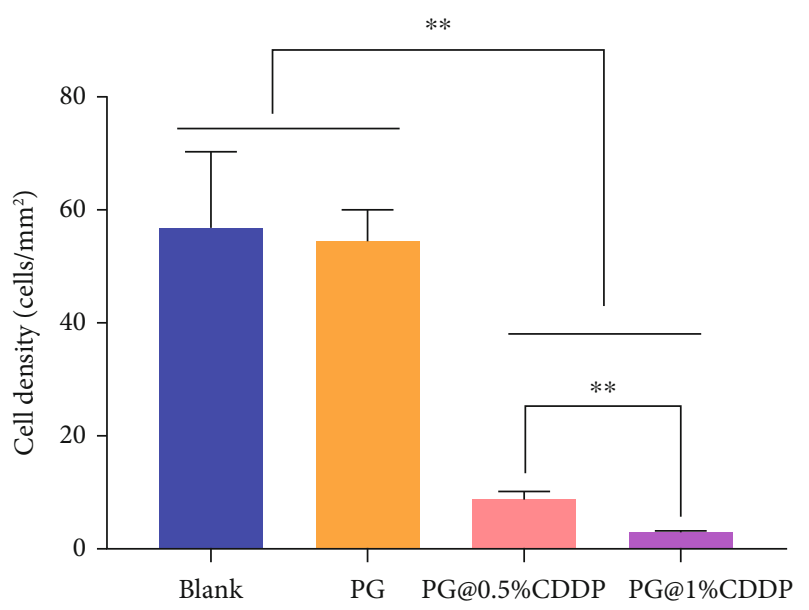

(b)

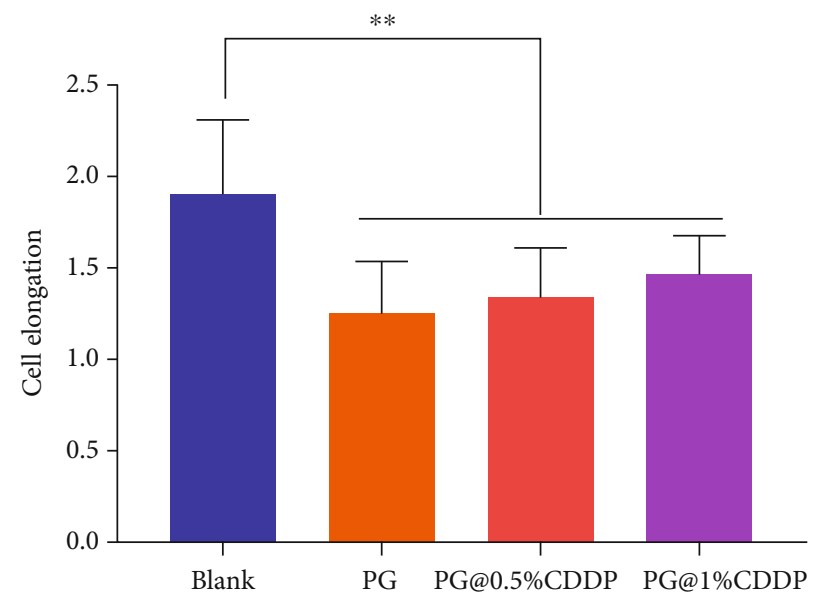

(c)

Figure 5: (a) Fluorescent images of blank, PG, PG@0.5\%CDDP, and PG@1\%CDDP fibers after $1 \mathrm{~d}$ of cell culture. (b) Cell density and (c) cell elongation on blank, PG, PG@0.5\%CDDP, and PG@1\%CDDP fibers.

In the PG group, the typical bands for peptide bonds from GT were found [31], e.g., amide-A band at $3200-3500 \mathrm{~cm}^{-1}$ corresponding to $\mathrm{N}-\mathrm{H}$ stretching vibrations, the amide II band corresponding to $1534 \mathrm{~cm}^{-1}$, and the amide III band corresponding to $1239 \mathrm{~cm}^{-1}$; the typical bands from PCL were found, e.g., the peak at $1700 \mathrm{~cm}^{-1}$ corresponding to $\mathrm{C}=\mathrm{O}$ asymmetric, the peak at $2920 \mathrm{~cm}^{-1}$ corresponding to $\mathrm{C}-\mathrm{H}$ asymmetric, and the peak at $2840 \mathrm{~cm}^{-1}$ corresponding to $\mathrm{C}-\mathrm{H}$ symmetric [32]. The spectra of PG@0.5\%CDDP and PG@1\%CDDP fibers include all of the characteristic peaks of PG. Particularly, the peak at $800 \mathrm{~cm}^{-1}$ in PG@0.5\%CDDP and PG@1\%CDDP fibers was detected, indicating that CDDP was successfully loaded into PG fibers.

The XRD patterns of CDDP and PG-based fibers are shown in Figure 3(b). The CDDP had obvious crystallization peaks between 10 and $20^{\circ}$ [33]. The PG group possessed characteristic diffraction peaks between 20 and $25^{\circ}[22,23]$. When PG was loaded with different concentrations of CDDP, the PG@0.5\%CDDP and PG@1\%CDDP fibers showed the characteristic diffraction peak of CDDP at 10$20^{\circ}$, and the typical diffraction peak of PG appeared between 20 and $25^{\circ}$, also suggesting that CDDP was successfully loaded into PG fibers.

An initial burst release of PG@0.5\%CDDP fibers was detected within the first $1 \mathrm{~h}$ ( $70 \%$ of the drug was released), followed by a sustained release profile (Figure 4). Similarly, there was an initial burst found in release in the PG@1\%CDDP fibers (77\% of the drug was released). A sus- tained release in PG@1\%CDDP fibers was observed with increasing the time from 0 to $12 \mathrm{~h}$.

3.2. Anticancer Behavior of PG-Based Composite Fibers. Cancer cell adhesion is the initial response to the microenvironment, which precedes other cellular behaviors, e.g., spreading, migration, and proliferation $[34,35]$. The composite fibers were tested in vitro for their anticancer behaviors against human prostate cancer cells. Initial cell response on all samples after $1 \mathrm{~d}$ was measured with a fluorescence staining of the actin cytoskeleton and nucleus. It was found that the PG fibrous films containing CDDP greatly affected the attachment and morphology of cancer cells (Figure 5(a)). With an increased amount of CDDP from 0 to $1 \%$, the number of adhered cells gradually decreased and the expression of the actin cytoskeleton (red) reduced.

To better understand the effect of PG@CDDP fibers on the initial behavior of cancer cells, cell density, elongation, and area per cell were quantified by the analysis of the positively stained cells. Figure 5(b) displays that the density of cancer cells significantly decreased with increasing the concentration of CDDP in PG fibers, indicating that the released CDDP from PG fibers greatly inhibited the attachment of cancer cells. Cell elongation is an essential morphological feature for the interaction between cells and biomaterials by mediating the cytoskeleton actin and stimulating the cell nucleus, affecting cell migration, viability, and proliferation $[36,37]$. Figure $5(c)$ shows that the cell elongation of PG- 


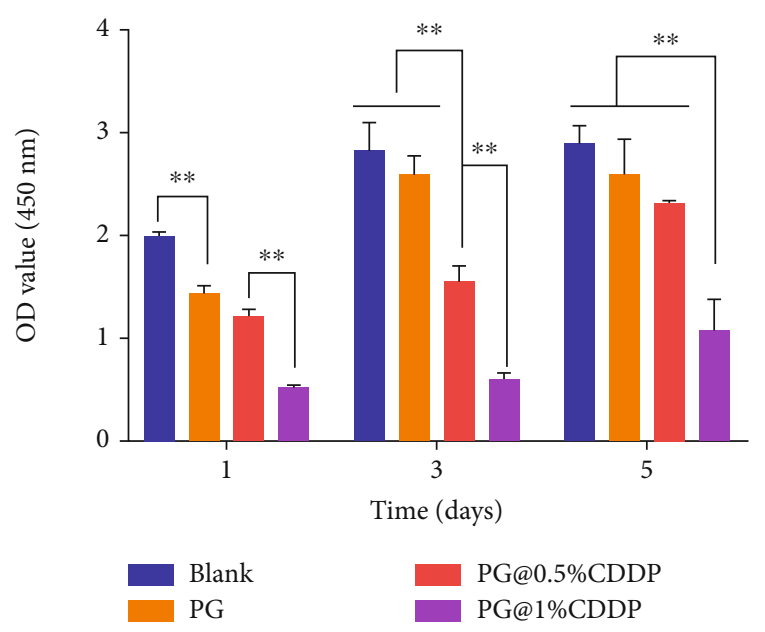

FIGURE 6: The viability of cancer cells on blank, PG, PG@0.5\%CDDP, and PG@1\%CDDP fibers after 1, 3, and 5d of cell culture.

based fibers was significantly reduced compared to that of the blank, which suggests that PG-based fibers could suppress the migration of cancer cells.

The CCK- 8 viability assay was performed after 1,3 , and $5 \mathrm{~d}$ of incubation. As shown in Figure 6, after $1 \mathrm{~d}$ of incubation, the $\mathrm{OD}$ value decreased with an increased concentration of CDDP in PG fibers, indicating that the addition of CDDP in PG fibers had a negative effect on the viability of cancer cells. After 3 and $5 \mathrm{~d}$ of incubation, the viability of cancer cells displayed a similar trend with that of $1 \mathrm{~d}$ of incubation. Taken together, the designed and prepared PG@CDDP fibers significantly inhibited cell attachment and viability, having great potential for the treatment of prostate cancer.

\section{Conclusions}

In summary, a PG@CDDP fibrous matrix was successfully fabricated using the electrospinning strategy. CDDP was introduced into the PG fibers based on the measurements of FT-IR spectroscopy and XRD. Small particles were found in PG@CDDP fibers compared to the PG fibers due to the addition of CDDP. The diameter of PG@1\%CDDP fibers slightly reduced compared to those of the PG and PG@0.5\%CDDP fibers. An initial burst release of PG@1\%CDDP fibers within the first $1 \mathrm{~h}$ was found, followed by a sustained release profile from 0 to $12 \mathrm{~h}$. The density, elongation, and viability of adhered cancer cells significantly decreased with increasing the concentration of CDDP in PG fibers. Therefore, the prepared PG@CDDP fiber matrix shows great potential for the treatment of prostate cancer.

\section{Data Availability}

The data used to support the findings of this study are available from the corresponding author upon request.

\section{Conflicts of Interest}

The authors declare no competing financial interest.

\section{Acknowledgments}

The authors are very grateful for the financial support by Key Research and Development Plan of Shandong Province (No. 2018GSF118127), the National Natural Science Foundation of China (Nos. 81602226, 81202016), Shandong Provincial Natural Science Foundation (Nos. ZR2015HQ002, ZR2020QH069), Shandong Province Medical and Health Science Technology Development Project (No. 2019WS464), and China Postdoctoral Science Foundation (Nos. 2016M590638, 2016M590641).

\section{References}

[1] V. Shanmugam, S. Selvakumar, and C.-S. Yeh, "Near-infrared light-responsive nanomaterials in cancer therapeutics," Chemical Society Reviews, vol. 43, no. 17, pp. 6254-6287, 2014.

[2] C. Wang, J. Yang, C. Dong, and S. Shi, "Glucose oxidaserelated cancer therapies," Advanced Therapeutics, vol. 3, article 2000110, 2020.

[3] J.-J. Wang, K.-F. Lei, and F. Han, "Tumor microenvironment: recent advances in various cancer treatments," European Review for Medical and Pharmacological Sciences, vol. 22, no. 12, pp. 3855-3864, 2018.

[4] H. Barabadi, K. Damavandi Kamali, F. Jazayeri Shoushtari et al., "Emerging theranostic silver and gold nanomaterials to combat prostate cancer: a systematic review," Journal of Cluster Science, vol. 30, no. 6, pp. 1375-1382, 2019.

[5] M. Barani, F. Sabir, A. Rahdar, R. Arshad, and G. Z. Kyzas, "Nanotreatment and nanodiagnosis of prostate cancer: recent updates," Nanomaterials, vol. 10, no. 9, p. 1696, 2020.

[6] J. E. McNeal, "Origin and development of carcinoma in the prostate," Cancer, vol. 23, no. 1, pp. 24-34, 1969.

[7] P. McGale, C. Taylor, C. Correa et al., "Effect of radiotherapy after mastectomy and axillary surgery on 10-year recurrence and 20-year breast cancer mortality: meta-analysis of individual patient data for 8135 women in 22 randomised trials," The Lancet, vol. 383, 2014.

[8] S. Gai, G. Yang, P. Yang et al., "Recent advances in functional nanomaterials for light-triggered cancer therapy," Nano Today, vol. 19, pp. 146-187, 2018.

[9] S. Mura, J. Nicolas, and P. Couvreur, "Stimuli-responsive nanocarriers for drug delivery," Nature Materials, vol. 12, no. 11, pp. 991-1003, 2013.

[10] E. Blanco, H. Shen, and M. Ferrari, "Principles of nanoparticle design for overcoming biological barriers to drug delivery," Nature Biotechnology, vol. 33, no. 9, pp. 941-951, 2015.

[11] J. Shi, P. W. Kantoff, R. Wooster, and O. C. Farokhzad, "Cancer nanomedicine: progress, challenges and opportunities," Nature Reviews. Cancer, vol. 17, no. 1, pp. 20-37, 2017.

[12] W. Qiao, B. Wang, Y. Wang, L. Yang, Y. Zhang, and P. Shao, "Cancer therapy based on nanomaterials and nanocarrier systems," Journal of Nanomaterials, vol. 2010, Article ID 796303, 2010.

[13] T. Okuda, K. Tominaga, and S. Kidoaki, “Time-programmed dual release formulation by multilayered drug-loaded nanofiber meshes," Journal of Controlled Release, vol. 143, no. 2, pp. 258-264, 2010.

[14] X. Xu, X. Chen, P. Ma, X. Wang, and X. Jing, "The release behavior of doxorubicin hydrochloride from medicated fibers prepared by emulsion-electrospinning," European Journal of 
Pharmaceutics and Biopharmaceutics, vol. 70, no. 1, pp. 165$170,2008$.

[15] X. Hu, S. Liu, G. Zhou, Y. Huang, Z. Xie, and X. Jing, "Electrospinning of polymeric nanofibers for drug delivery applications," Journal of Controlled Release, vol. 185, pp. 12-21, 2014.

[16] J. Xue, M. He, H. Liu et al., "Drug loaded homogeneous electrospun PCL/gelatin hybrid nanofiber structures for antiinfective tissue regeneration membranes," Biomaterials, vol. 35, no. 34, pp. 9395-9405, 2014.

[17] R. Qi, R. Guo, F. Zheng, H. Liu, J. Yu, and X. Shi, "Controlled release and antibacterial activity of antibiotic-loaded electrospun halloysite/poly(lactic-co-glycolic acid) composite nanofibers," Colloids Surfaces B Biointerfaces, vol. 110, pp. 148-155, 2013.

[18] B. Song, C. Wu, and J. Chang, "Dual drug release from electrospun poly(lactic-co-glycolic acid)/mesoporous silica nanoparticles composite mats with distinct release profiles," Acta Biomaterialia, vol. 8, no. 5, pp. 1901-1907, 2012.

[19] X.-Z. Sun, G. R. Williams, X.-X. Hou, and L.-M. Zhu, "Electrospun curcumin-loaded fibers with potential biomedical applications," Carbohydrate Polymers, vol. 94, no. 1, pp. 147-153, 2013.

[20] G. Xia, H. Zhang, R. Cheng et al., "Localized controlled delivery of gemcitabine via microsol electrospun fibers to prevent pancreatic cancer recurrence," Advanced Healthcare Materials, vol. 7, no. 18, p. 1800593, 2018.

[21] S. Chen, S. K. Boda, S. K. Batra, X. Li, and J. Xie, "Emerging roles of electrospun nanofibers in cancer research," Advanced Healthcare Materials, vol. 7, no. 6, p. 1701024, 2018.

[22] Q. Zhou, H. Zhang, Y. Zhou et al., “Alkali-mediated miscibility of gelatin/polycaprolactone for electrospinning homogeneous composite nanofibers for tissue scaffolding," Macromolecular Bioscience, vol. 17, pp. 1-10, 2017.

[23] B. Feng, H. Tu, H. Yuan, H. Peng, and Y. Zhang, "Acetic-acidmediated miscibility toward electrospinning homogeneous composite nanofibers of GT/PCL," Biomacromolecules, vol. 13, no. 12, pp. 3917-3925, 2012.

[24] W. Shen, X. Chen, J. Luan, D. Wang, L. Yu, and J. Ding, "Sustained codelivery of cisplatin and paclitaxel via an injectable prodrug hydrogel for ovarian cancer treatment," ACS Applied Materials \& Interfaces, vol. 9, no. 46, pp. 40031-40046, 2017.

[25] A. Sherif, L. Holmberg, E. Rintala et al., "Neoadjuvant cisplatinum based combination chemotherapy in patients with invasive bladder cancer: a combined analysis of two Nordic studies," European Urology, vol. 45, no. 3, pp. 297-303, 2004.

[26] G. Yang, X. Li, Y. He, J. Ma, G. Ni, and S. Zhou, "From nano to micro to macro: electrospun hierarchically structured polymeric fibers for biomedical applications," Progress in Polymer Science, vol. 81, pp. 80-113, 2018.

[27] H. Chen, X. Huang, M. Zhang et al., "Tailoring surface nanoroughness of electrospun scaffolds for skeletal tissue engineering," Acta Biomaterialia, vol. 59, pp. 82-93, 2017.

[28] Q. Zhou, J. Xie, M. Bao et al., "Engineering aligned electrospun PLLA microfibers with nano-porous surface nanotopography for modulating the responses of vascular smooth muscle cells," Journal of Materials Chemistry B, vol. 3, no. 21, pp. 4439-4450, 2015.

[29] Q. Zhang, Y. Ji, W. Zheng et al., "Electrospun nanofibers containing strontium for bone tissue engineering," Journal of Nanomaterials, vol. 2020, Article ID 1257646, 2020.
[30] E. Ortiz-Islas, M. E. Manríquez-Ramírez, A. Sosa-Muñoz et al., "Preparation and characterisation of silica-based nanoparticles for cisplatin release on cancer brain cells," IET Nanobiotechnology, vol. 14, no. 3, pp. 191-197, 2020.

[31] I. Rajzer, E. Menaszek, R. Kwiatkowski, J. A. Planell, and O. Castano, "Electrospun gelatin/poly( $\varepsilon$-caprolactone) fibrous scaffold modified with calcium phosphate for bone tissue engineering," Materials Science \& Engineering. C, Materials for Biological Applications, vol. 44, pp. 183-190, 2014.

[32] A. Khalf and S. Madihally, "Modeling the permeability of multiaxial electrospun poly( $\varepsilon$-caprolactone)-gelatin hybrid fibers for controlled doxycycline release," Materials Science \& Engineering. C, Materials for Biological Applications, vol. 76, pp. 161-170, 2017.

[33] U. Aggarwal, A. K. Goyal, and G. Rath, "Development and characterization of the cisplatin loaded nanofibers for the treatment of cervical cancer," Materials Science \& Engineering. C, Materials for Biological Applications, vol. 75, pp. 125-132, 2017.

[34] U. Cavallaro and G. Christofori, "Cell adhesion and signalling by cadherins and Ig-CAMs in cancer," Nature Reviews. Cancer, vol. 4, no. 2, pp. 118-132, 2004.

[35] G. Jacquemet, H. Hamidi, and J. Ivaska, "Filopodia in cell adhesion, 3D migration and cancer cell invasion," Current Opinion in Cell Biology, vol. 36, pp. 23-31, 2015.

[36] Q. Zhou, J. Chen, Y. Luan et al., "Unidirectional rotating molecular motors dynamically interact with adsorbed proteins to direct the fate of mesenchymal stem cells," Science advances, vol. 6, article eaay2756, 2020.

[37] Y. Jia, W. Yang, K. Zhang et al., "Nanofiber arrangement regulates peripheral nerve regeneration through differential modulation of macrophage phenotypes," Acta Biomaterialia, vol. 83, pp. 291-301, 2019. 